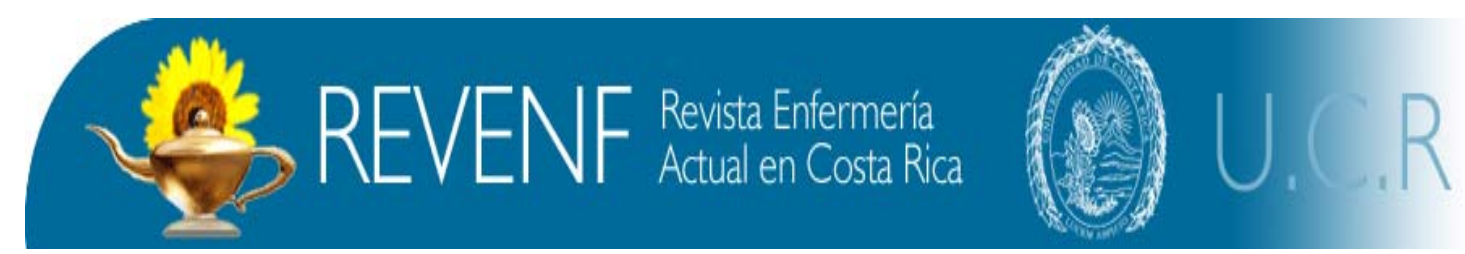

Revista Semestral Número 18 Abril - Setiembre 2010

ISSN 1409-4568

\title{
DISEÑO DE UNA GUIA DIRIGIDA A LOS Y LAS ENCARGADAS DEL CUIDADO EN EL HOGAR, DE MENORES CON DISCAPACIDAD MOTORA ${ }^{1}$
}

\section{COMO CITAR ESTE ARTÍCULO}

García L, Esteban; Muñoz C. Pablo; Porras H. Stephanie; Leiva D. Viriam. Diseño de una guía dirigida a los y las encargadas del cuidado en el hogar, de menores con discapacidad motora. Rev. Enfermería Actual en Costa Rica [en línea].2010, No.18 [citado (fecha)]. Disponible World Wide Web: <http://www.revenf.ucr.ac.cr/guiadiscapacidad.pdf> ISSN 1409-4568

\author{
Esteban Alonso García Leiva ${ }^{2}$ \\ Juan Pablo Muñoz Campos ${ }^{3}$ \\ Stephanie Porras Hidalgo ${ }^{4}$ \\ Viriam Leiva Díaz
}

Institución: Escuela de Rehabilitación de Costa Rica

\section{RESUMEN}

En el siguiente artículo se presentan los resultados obtenidos con la ayuda de una guía que fue creada para ayudar a aquellas personas que cuidan, en su hogar, a niños, niñas y/o adolescentes que poseen alguna discapacidad motora; tal instrumento constituye un medio educativo con el cual se facilita, a los encargados de estos menores, el proceso de intervención y recuperación. La elaboración de dicha guía se elaboró a partir de un proceso diagnóstico de las necesidades de orientación de los/as encargados/as del cuidado de personas menores con discapacidad, también se tomó en cuenta a las maestras y al equipo interdisciplinario que labora en la Escuela de Rehabilitación de Costa Rica. Esta guía se construyó de acuerdo con la clasificación de las necesidades identificadas según los requisitos universales planteados por Dorothea Orem en su Teoría de Autocuidado. Posteriormente, la guía fue validada por expertos y por la población meta con el fin de corregirla y presentar la versión final.

Palabras clave: Enfermería - Cuidado - Cuidador -- Discapacidad - Niñez - Adolescencia.

\section{ABSTRACT}

\footnotetext{
${ }^{1}$ Fecha de recepción: Febrero 2009

Fecha de aceptación: Marzo 2010

${ }^{2}$ Enfermero, HomeWatch Caregivers Latinoamerica. Correo electrónico: estebanagl@gmail.com

${ }^{3}$ Enfermero, Hospital Calderón Guardia. Correo electrónico: juanpablomc@gmail.com

${ }^{4}$ Enfermera, Hospital Clínica Católica. Correo electrónico: sph1914@yahoo.com

${ }^{5}$ Docente de la Escuela de Enfermería, Universidad de Costa Rica. Enfermera. Psicóloga. Magistra en

Psicopedagogía. Correo electrónico: viriaml@gmail.com
} 


\section{A GUIDE DESIGN FOR THE HOME CARE RESPONSIBLE OF CHILDREN WITH MOTOR DISABILITIES}

This article presents the results obtained of the creation of a guide directed to the person responsible of the child or adolescent with motor disability, who also suffer from illness in their homes. This guide is the educational and informative tool for parents, family members, and third parties, which makes easier the intervention and recovery of the children under their care. This guide was made from a diagnostic process of the need of orienting the people who take care of children with disabilities and also of guiding the teachers and the interdisciplinary team who are in charge of Rehabilitation School. The guide was built classifying the identified needs according to universal conditions made by Dorothea Orem in her Theory of Self-Care. Next, the guide was evaluated by experts and by the people to whom this manual is intended, correcting and presenting the final version.

Keywords: Nursery - Care - Disability - Guardian -Child - Adolescent - Theory of Self-Care.

\section{INTRODUCCIÓN}

A partir del estudio que se presenta a continuación, se construyó una guía destinada a todas aquellas personas que se encargan de niños, niñas y/o adolescentes con alguna discapacidad motora. La población beneficiada asiste a la Escuela de Rehabilitación de Costa Rica.

El estudio nació una vez que se realizó un diagnóstico de las necesidades de orientación de las personas cuidadoras principales, maestras y del equipo interdisciplinario, con esta información se construyó un instrumento educativo, comprensible y sin fines de lucro con el que se puede responder a muchas interrogantes relacionadas con el cuido de la población mencionada.

En relación con esta temática, hay que rescatar los datos de la Organización Mundial de la Salud (OMS) del año 2002, los cuales indican que de un 7 a un $10 \%$ de la población mundial tiene algún tipo de discapacidad ya sea física, sensorial y/o mental.
Asimismo, en Costa Rica, según los datos del Instituto Nacional de Estadística y Censos (INEC, 2001), la población nacional infantil y adolescente está constituida por 1608 870 personas, de ellas, 38437 tienen algún tipo de discapacidad. Además, es importante destacar que el país cuenta con legislación relacionada con la igualdad de oportunidades para las personas con discapacidad (Ley 7600): esto representa un marco legal que plantea la igualdad entre personas con discapacidad y personas sin discapacidad y, a su vez, declara que el desarrollo integral es una temática de interés público.

Por tanto, teniendo en cuenta la cantidad de personas involucradas con la discapacidad y el marco legal establecido, se justifica que los profesionales en salud tengan los conocimientos necesarios para brindar una atención integral a esta población. A su vez, es latente la necesidad de contar con herramientas sistematizadas para cumplir con dicho propósito. En este punto cabe destacar la escasez de este tipo de instrumentos, por tanto, es inminente la creación de los mismos. 
Del anterior planteamiento nace el objetivo que guió la presente investigación, el cual fue desarrollar una modalidad de atención, con el uso de una guía dirigida a personas que se encargan, en su hogar, de menores con discapacidades motoras.

Al mismo tiempo, se plantearon los siguientes objetivos específicos:

1. Caracterizar la población participante en el estudio.

2. Efectuar un diagnóstico de las necesidades de orientación de los/as encargados/as del cuidado de la población mencionada.

3. Construir una guía de atención, dirigida al/a encargado/a del cuidado, basada en el diagnóstico.

4. Implementar un proceso de validación de la guía de atención.

5. Construir la guía definitiva sustentándose en la validación.

Entre de los antecedentes se encuentran, en el ámbito internacional, el estudio realizado por Gaite, Cantero, González y García (2005), llamado Necesidades de las y los pacientes pediátricos con enfermedades raras y sus familias en Cantabria. Estos investigadores concluyeron que la identificación de las necesidades de esta población, y la asistencia que se les brinda, presentan deficiencias en cuanto a su sistematización.

Asimismo, en Costa Rica, existe un estudio realizado por Pampillo (1995), relacionado con las necesidades de las personas con discapacidades múltiples y/o severas; la autora recalca que la falta de capacitación en los padres y las madres representa la mayor limitación para el manejo de hijos e hijas con discapacidad; por otra parte, recomienda diseñar una guía práctica y funcional para brindar respuesta a las necesidades diagnosticadas.
Espinoza, Mena y Salas (2004) en su estudio respecto de la calidad de vida de niños y niñas con discapacidad por enfermedad crónica, destacan que los padres y las madres manejan muy mal el estrés, algunos sobreprotegen a sus hijos e hijas y, muchas veces, las madres son quienes se encargan de cuidar a los/as menores.

A su vez, Leal (2005), en el área de los estudios interdisciplinarios acerca de la discapacidad, propone la creación de una guía para sistematizar información ligada a la parálisis cerebral con el fin de aclarar dudas e inquietudes para resolver problemas cotidianos.

Finalmente, en el área de Enfermería, se destaca el estudio efectuado por Arce, Barrientos, Castillo y Varela (2006), el cual tuvo lugar en la Escuela de Rehabilitación en Costa Rica; ellos indican que la intervención de Enfermería favorecerá el desarrollo físico, psicosocial y cognitivo del niño, la niña y el/la adolescente con discapacidad. Este estudio representa el primer acercamiento -desde la Enfermería- en la institución y constituyó un aliciente para futuras investigaciones.

\section{MATERIALES Y MÉTODOS}

En cuanto al proceso metodológico, el estudio se basó en el modelo mixto de investigación el cual, según Hernández, Fernández-Collado y Baptista (2006) es un proceso que recolecta, analiza $y$ vincula datos cuantitativos $y$ cualitativos en un mismo estudio o una serie de investigaciones (p: 755); el diseño de investigación es de tipo acción-práctico cuya finalidad, según Álvarez-Gayou, mencionado por Hernández et al (2006), es resolver problemas cotidianos y mejorar prácticas concretas. 
Es importante enmarcar el lugar de la investigación, por tanto, a continuación aparece una breve reseña del mismo. La Escuela de Rehabilitación de Costa Rica fue creada en 1972 con el objetivo de brindar una atención integral a los niños, las niñas, y los/as adolescentes con problemas neuro-músculoesqueléticos. Esta población en su mayoría se ve afectada por una parálisis cerebral.

En la actualidad, la Escuela de Rehabilitación brinda una educación fundamentada en un sistema de niveles que debe ser cursado, por las personas con discapacidad, hasta que egresen de la institución. Dichos niveles son: estimulación temprana, maternal, kinder, primer ciclo, segundo ciclo, tercer ciclo y cuarto ciclo. Además, cuenta con un equipo interdisciplinario encargado de realizar visitas de seguimiento a los hogares de algunos/as jóvenes estudiantes que no pueden asistir regularmente a lecciones.

Respecto de la población en estudio, ésta estuvo constituida por las y los encargados de cuidar a los/as menores, con discapacidad motora, matriculados en la Escuela de Rehabilitación. Los criterios de selección fueron los siguientes:

Ser cuidador o cuidadora principal.

Que el niño, la niña y/o el/la, a su cargo, haya presentado, al menos, un proceso mórbido que le impida asistir regularmente- a lecciones.

Para efectos de la aplicación del cuestionario diagnóstico, se requiere que la persona con discapacidad estuviese cursando entre el primer y el cuarto ciclo ya que es la población que ha experimentado más dificultades e relación con el cuidado.
Estar anuente a participar en el estudio. Para ello se solicitó el consentimiento de las personas participantes.

A su vez, se entenderá como niño o niña a aquellas personas que, según la ley 7739 de Costa Rica, denominada Código de la Niñez y Adolescencia (1998), se encuentren entre los 0 y 12 años de edad; adolescente es aquella persona que, según la misma ley, se encuentra entre los 13 y 18 años de edad. Sin embargo, respecto del desarrollo humano, se destaca que: la adolescencia va terminando gradualmente con el comienzo de la vida adulta, alrededor de los 18 - 20 años (Rodríguez, 1995; p. 101). Por lo anterior, se decidió incluir a los encargados y encargadas del cuidado de alguna persona cuya edad se encuentre en ese rango.

Los instrumentos de recolección de datos están compuestos por 3 cuestionarios diagnósticos dirigidos respectivamente- a los encargados y las encargadas de la atención de los niños, las niñas, los adolescentes y las adolescentes con discapacidad; también está dirigido a los/as las educadores/as de la Escuela de Rehabilitación y al equipo interdisciplinario de la institución que realiza las visitas al hogar.

Dichos instrumentos diagnósticos fueron creados para recopilar los datos necesarios con los cuales se diagnosticaron las necesidades de orientación que poseen las personas cuidadoras principales. Además, se aplicó un cuestionario para evaluar los talleres de validación de la guía de atención; dicho instrumento fue aplicado a las personas participantes encargadas del cuidado.

Para elaborarlo se utilizaron como base los requisitos universales planteados por Dorothea Orem en su Teoría del Autocuidado (1983). 
El desarrollo del estudio se dividió en 6 etapas:

Primera Etapa. Introducción al Campo de Trabajo: En dicha etapa se realizó una pasantía en la Escuela de Rehabilitación con el objetivo de orientar a los/as investigadores/as en la temática de la discapacidad; igualmente, se busca un mayor involucramiento con las actividades que se realizan en la institución.

Segunda Etapa. Coordinaciones: En ésta se llevaron a cabo las coordinaciones requeridas para el desarrollo de la investigación. Se destacan las que fueron realizadas junto a las entidades de la institución destinadas a los espacios de aplicación de los cuestionarios diagnósticos y a los espacios para la ejecución de los talleres de validación. También se llevaron a cabo, como parte de la validación déla guía, coordinaciones de citas con especialistas pertenecientes a las áreas de pediatría, psicopedagogía y discapacidad.

Tercera Etapa. Trabajo de Campo: Esta etapa comprendió el proceso diagnóstico, es decir, la aplicación de los cuestionarios y el análisis de datos. De esta manera, de acuerdo con los criterios de selección de la población, se contó con la participación de 18 personas cuidadoras que respondieron el instrumento correspondiente. Asimismo, se contó con la participación de 9 docentes de la institución (una de cada nivel y del kinder) y con la participación de la terapeuta física de la institución (como miembro del equipo interdisciplinario.

Posteriormente, se realizó una sistematización y análisis de los datos cuantitativos y cualitativos obtenidos, luego, se delimitaron las necesidades de orientación encontradas para consensuar las que fueran comunes a la mayoría de personas implicadas en el estudio, para así poder determinar las necesidades de la población.

Cuarta Etapa. Diseño de la guía de Atención: En esta etapa, a partir de los resultados obtenidos en el diagnóstico de las necesidades de orientación de la población, se construye el primer borrador de la guía de atención.

Quinta Etapa. Validación: El proceso de validación de la guía se efectuó en 2 fases. La primera de ellas corresponde a la revisión de la guía hecha por los expertos de las áreas de pediatría, psicopedagogía y discapacidad, quienes brindaron recomendaciones pertinentes acerca de las correcciones que se le debía realizar a la guía para clarificar sus contenidos. La segunda fase de la validación se llevó a cabo mediante talleres estructurados a partir de los contenidos de la guía; en dichos talleres participaron personas cuidadoras que cumplían los criterios de selección; la finalidad era verificar que los participantes comprendiesen la guía.

Sexta Etapa. Construcción de la Guía Definitiva: En esta etapa se construyó la versión final de la guía una vez aprobada la validación. Con esta guía se da respuesta a las distintas dudas relacionadas con la orientación de las personas encargadas del cuidado. Esta guía es accesible para la mayor cantidad de interesados posibles, por tanto, no contempla algún fin de lucro.

Entre las consideraciones éticas, contempladas en esta investigación, se encuentran: el principio de beneficencia ya que las personas participantes del estudio obtuvieron información valiosa acerca del cuidado de Enfermería que puede brindarse a las personas menores con discapacidad; se aseguró absoluta confidencialidad y fidelidad de la 
información brindada; se respetó, igualmente, el principio de justicia con el cual se asegura a los participantes el que no renuncien a una cantidad excesiva de tiempo que les genere algún inconveniente. $\mathrm{La}$ presente investigación se apegó totalmente al principio de veracidad en cuanto a las personas participantes y a los resultados obtenidos. Finalmente, hay que indicar que la participación de los y las cuidadoras principales fue totalmente voluntaria: se les explicó la naturaleza y finalidad de la investigación, de esta forma, se logró el consentimiento de las mismas.

\section{RESULTADOS}

Los resultados obtenidos se integran a partir de tres aspectos: a) los resultados obtenidos durante la aplicación de cuestionarios diagnósticos en la etapa de trabajo de campo; b) la construcción de la guía de atención y c) el proceso de validación.

Los hallazgos del proceso diagnóstico se plantean a partir de los requisitos de autocuidado propuestos por $\underline{\text { Orem }}$ (1983) y las necesidades de orientación de las personas encargadas de la atención en relación con el cuidado.

De las 18 personas cuidadoras, 17 corresponden a mujeres cuyas edades se encuentran entre los 27 y 65 años; en cuanto a la relación de parentesco que mantienen con el/la menor que cuidan, la mayoría (15) de ellas son madres del/a infante; respecto de la ocupación que ejercen, en su mayoría son amas de casa.

En cuanto al estado civil, 8 de ellas son solteras o divorciadas y el resto casadas.

En cuanto al género de quienes reciben los cuidados se tiene que: 11 son hombres, 6 mujeres y de la persona restante no se especificó su género. La edad de esta última población oscila entre los 10 y 19 años. En cuanto a la causa que generó la discapacidad, se tiene que, en 15 de los casos, se debe a una parálisis cerebral que se dio entre el nacimiento y el período neonatal.

Respecto de las necesidades de ingesta de alimentos, siete de personas las personas que reciben cuidado, utilizan algún dispositivo, principalmente del tipo sonda de gastrostomía, para facilitar la ingesta de alimentos.

Asimismo, al consultar algunos aspectos relacionados con el requisito universal de aire, los encuestados indican que las enfermedades respiratorias son las que producen un mayor ausentismo en las escuelas, entre las más comunes están: cuadros gripales, neumonía, bronconeumonía, bronquitis y asma bronquial. Además agregaron que, para mejorar la condición respiratoria las personas menores con discapacidad, requieren diariamente el uso de técnicas para el manejo de secreciones (percusión y drenaje postural) así como de dispositivos para aspirar secreciones $\mathrm{y}$ diferentes fuentes de oxígeno.

En cuanto al requisito universal de eliminaciones vesicales e intestinales, en el primer caso, ninguna persona indica que el niño, la niña o adolescente que cuida requiere la utilización de dispositivos para favorecer la orina. En el segundo caso, se consultó si utilizaban algún tipo de laxantes método para facilitar la eliminación fecal: el $50 \%$ de la población de personas cuidadoras los emplea.

Al consultar a los encargados del cuidado respecto conocimiento que poseían en relación con la técnica del masaje colónico y la reflexología como alternativa del manejo del estreñimiento 
crónico, 5 personas indican conocerlas, sin embargo, al solicitarles que las describieran fueron bastantes imprecisos. Nadie se refirió a la reflexología.

En relación con la permanencia en cama de los niños, niñas y adolescentes durante los períodos de enfermedad, diez personas cuidadoras corroboran que los/as infantes $\mathrm{y}$ adolescentes suelen permanecer en cama si se da alguna patología.

Por otra parte, y con el fin de identificar patrones similares a los presentados en el síndrome del cuidador, se indagó acerca de diferentes aspectos asociados al mismo y su aparición en los/as encargados/as del cuido, se tiene que: 15 personas indican haber presentado problemas de salud en el último año, entre las principales patologías padecidas están cefaleas, afecciones músculo esqueléticas, fatiga e insomnio (en más de 11 personas). Esta situación según las personas cuidadoras se debe a un exceso de labores en el hogar ya que el cuido de la persona con discapacidad representa un gran esfuerzo físico. Por otra parte, un tercio de la población menciona que el cuido -en algún momento- ha sobrepasado sus capacidades.

Aunado a los datos anteriores, las personas cuidadoras mencionan que no reciben suficiente colaboración para llevar a cabo la atención de la persona con discapacidad y, en 17 de los casos, la jornada de trabajo es de hasta 24 horas.

Respecto de las necesidades de orientación encontradas en las personas cuidadoras, los grupos consultados indican que hay una variedad importante de requerimientos tales como: el manejo de equipo $\mathrm{y}$ dispositivos la administración y uso de medicamentos, las acciones en caso de que se presenten convulsiones, manejo de secreciones, el uso de oxígeno, la higiene, los cambios posturales, el baño en cama, primeros auxilios, características de la adolescencia, diferentes aspectos de la sexualidad de la persona con discapacidad.

\section{DISCUSIÓN}

En la población de personas cuidadoras se observó una feminización del cuidado. De acuerdo García, M., Mateo, I., y Maroto, G (2004), el rol de la persona cuidadora no es equitativa en las familias y la feminización del mismo pone de manifiesto las cargas diferenciales del cuidado. Por ello género, convivencia y parentesco son las variables más importantes de cara a predecir qué persona del núcleo familiar va a ser la cuidadora principal (s.p.).

Es importante tomar en cuenta el estado civil de los/as participantes: la mayoría de las mujeres son solteras o divorciadas cuya familia es de tipo uniparental. De lo anterior se concluye que la jefatura del hogar está ejercida exclusivamente por ellas en para la comprensión factor que perjudica y aumenta el número de responsabilidades con las que tiene que lidiar la persona encargada del cuidado. Cabe agregar que estas encargadas además cumplen el rol de proveedoras.

El origen de la discapacidad motora -de los menores que reciben el cuidado- se debe a una parálisis cerebral sufrida entre el nacimiento y el período neonatal. De acuerdo con Póo (2008), las complicaciones que se presentan con mayor frecuencia, producto de la parálisis cerebral, son las ortopédicas, las que se dan a nivel de tracto digestivo y las que se dan en el sistema 
respiratorio. Si tomamos en cuenta lo anterior y la edad en la que se dio la discapacidad, se puede concluir que estas poblaciones requieren conocer el sistema sustentador educativo expuesto por Orem (1983) para que la persona cuidadora implemente las medidas relacionadas con el autocuidado universal 0 las derivaciones terapéuticas, con el fin de mejorar la calidad de vida de las personas menores con discapacidad.

Tomando en cuenta los requisitos de autocuidado, específicamente la ingesta de alimento, agua y aire, es evidente que más de la tercera parte de los niños, las niñas o adolescentes que reciben cuidados requieren de algún dispositivo, principalmente del tipo sonda de gastrostomía, para facilitar la ingesta de alimentos. Al respecto Póo (2008) indica que entre las alteraciones gastrointestinales más frecuentes, en personas con parálisis cerebral, se encuentran el reflujo gastroesofágico y dificultades para la alimentación tales como la deglución; tales obstáculos obligan -a estos pacientes- a utilizar dispositivos para alimentarse $\mathrm{y}$, por tanto y, a su vez, es necesario que quienes los/as cuidan sean capacitados para tal tarea con el fin de que conozcan acerca de aplicación de medidas antirreflujo y de cuidados generales para, entre otras cosas, asegurar el uso de la sonda de gastrostomía u otro mecanismo de alimentación y para realizar licuados artesanales.

En cuanto al requisito universal de aire, las personas menores con discapacidad requieren (para mantener una buena condición respiratoria) conocer técnicas para el manejo de secreciones, saber acerca de dispositivos utilizados para aspirar secreciones y deben conocer el uso de fuentes de oxígeno; estos datos coinciden con lo indicado por Kozier, Erb y Olivieri (1993) quienes consideran que las secreciones, en personas con discapacidad motora, son una respuesta fisiológica debido a la reducción de la movilidad y aparición de los procesos patológicos. Por otra parte, Del Águila y Áibar (2006) indican que el reflujo es un padecimiento que también genera afección respiratoria causada por neumonías recurrentes secundarias, por tal razón, hay que aplicar medidas antirreflujo y técnicas adecuadas de alimentación para reducir la posibilidad de complicaciones respiratorias.

En cuanto al requisito universal de eliminaciones vesicales e intestinales, $y$ a pesar de que las personas cuidadoras afirmaron que ningún niño, niña o adolescente que cuidan requiere la utilización de dispositivos (cateterismo vesical intermitente o sonda vesical fija) para favorecer la salida de la orina se decidió incluirlo en la guía de atención, por lo expuesto por Harbin, Behrman, y Kliegman (1997), quienes indican que las dificultades urinarias, específicamente del tipo vejiga neurógena, son comunes en personas con alteración neurológica y patologías representativas de la discapacidad de tipo motor. A su vez, tanto la terapeuta ocupacional como las educadoras de la Escuela de Rehabilitación exponen que el uso de dispositivos como las sondas son una necesidad prioritaria de orientación en la persona cuidadora.

Tomando en cuenta las eliminaciones de tipo intestinal, es importante tomar en cuenta que las personas con discapacidad de tipo físico, o que presentan problemas de movilización, suelen padecer de estreñimiento (Fritz 2001). Para amortiguar los efectos del estreñimiento, las personas suelen usar laxantes puesto que éstos son fáciles de adquirir y pueden ser administrados sin prescripción médica. Debido a lo anterior, es necesario que los/as 
cuidadores/as reciban capacitación en cuánto a cómo y cuáles laxantes puede administrar. Esta propuesta fue implementada junto a otras indicaciones como el aplicar las técnicas de masaje colónico y de reflexología cuyo aprendizaje y ejecución adecuada pueden disminuir las dificultades de evacuación intestinal. Igualmente, se incluyeron recomendaciones de tipo nutricional.

Entre las razones que provocan gran ausentismo escolar se pueden citar las alteraciones gastrointestinales y las convulsiones. En relación con lo anterior, es necesario recalcar que una de las personas que cuidan posee un manejo deficiente al respecto. De igual forma, las cirugías agravan esta situación debido a que requiere de cuidados postoperatorios.

Es importante analizar el tiempo en el que el niño, niña o adolescente permanecen en cama dado que podrían derivarse algunas consecuencias como aumento en la dificultad para llevar a cabo los procesos respiratorios, problemas para realizar la evacuación intestinal, también pueden aparecer úlceras por presión. Por otra parte, hay que realizar el aseo general en cama, hay que aplicar la movilización pasiva y utilizar técnicas de mecánica corporal por situaciones de crecimiento corporal puesto que la población en estudio supera los diez años de edad. No se tomó en cuenta estas necesidades coinciden o no propiamente con la situación patológica.

Estas variables pueden aumentar las demandas de un agente de cuidado, por ende, precisan una mayor orientación para las personas cuidadoras ya que, como menciona Orem (1983) y requieren una mayor intervención de terapéutica desde la Enfermería siempre y cuando el padre, la madre, el tutor o la tutora de un niño o una niña presenten serias dificultades para mantener la cantidad o calidad de un cuidado. Por ello, es indispensable un instrumento de consulta constante, como el elaborado, para que las personas cuidadoras puedan enfrentar y manejar más adecuadamente este tipo de situaciones.

Algunos aspectos fundamentales tomado en cuenta, y relacionados con la fue salud de la persona cuidadora, fueron la presencia de padecimientos como cefaleas, afecciones músculoesqueléticas, fatiga e insomnio; todas estas afecciones están relacionadas con el exceso de labores en el hogar y el esfuerzo físico que representa el cuido de una persona con discapacidad (responden al síndrome del cuidador). Según Zambrano y Ceballos (2007), tanto los familiares que se encargan de la atención de una persona como los profesionales que brindan atención directa pueden manifestar dificultades, malestar físico y mental, incluso trastornos mentales, que tienen estrecha relación con su actividad como cuidadores (s.p.). Estas dificultades, a su vez, van a agravarse por la presencia de tristeza y estrés y sensación de carga - denominada síndrome del cuidador. En la mayoría de las ocasiones, el estrés rebasa la capacidad de afrontamiento que posee la persona encargada del cuidado (Zambrano y Ceballos, 2007). Las personas encuestadas afirman pasar por tal situación, por ende, necesitan de orientación al respecto.

Aunado a lo expuesto anteriormente, las personas cuidadoras mencionan que no reciben suficiente colaboración para atender a la persona con discapacidad; esto es compatible con lo expuesto por García, Mateo, y Maroto (2004) quienes mencionan que la duración de la 'jornada laboral' de una cuidadora no tiene principio ni fin y esta labor es tan 
sólo una de tantas que debe enfrentar durante su faena diaria.

Todo lo mencionado hasta el momento es fundamental para desarrollar la intervención de Enfermería desde el sistema definido por Orem (como el sustento teórico), de manera que la guía permite orientar y educar a los padres, madres, cuidadores o cuidadoras en situaciones específicas. Desde esta perspectiva, en la medida en que el sistema sustentador educativo logre satisfacer no sólo los intereses directos de los y las usuarias sino las necesidades de orientación de los y las cuidadoras, se esperaría una menor frecuencia de complicaciones ya que los y las cuidadoras implementarían en el sistemas de compensación parcial o total acciones intencionales (Orem, 1983) dirigidas al cuidado y autocuidado de los miembros dependientes basados en el conocimiento y la capacitación idónea (Benjumea, 2007).

Durante la etapa de Diseño de la Guía de Atención se realizó una selección de temas imprescindibles dentro del contenido de la guía. Seguidamente, se planteó la estructura temática que seguiría la guía la cual fue establecida en dos secciones: la primera está relacionada con Aspectos del cuidado del niño, la niña o adolescente con discapacidad motora, dividido a su vez en quince capítulos distribuidos con un orden secuencial para facilitar la lectura y comprensión por parte del usuario final. La segunda sección se concentró en El cuidador o la cuidadora principal y contempla el autocuidado de éstos últimos en un capítulo único que resume el abordaje de factores trascendentales, relacionados con la temática, encontrados en el diagnóstico.

Luego se continuó con la búsqueda bibliográfica correspondiente y se incorporó información didáctica que fuese comprensible para el público en general. En cuanto a este punto, la construcción de la guía fue más allá de la inclusión de información dado que además- incorporó mecanismos para hacerla atractiva como la adición de ayudas visuales tales como dibujos $\mathrm{y}$ las guías de lectura, constituidas por colores que diferenciaban cada capítulo; además se implementó el uso de preguntas generadoras, para conducir a la persona durante la lectura y la inclusión de recordatorios -separados del texto principal- para enriquecer la comprensión.

Seguidamente, se pasó a la etapa de validación por expertas. En esta etapa se recibieron aportes para mejorar la forma del modelo inicial, por tanto, colaboraron Enfermeras especialistas en Pediatría, Discapacidad y Psicopedagogía; por sus observaciones se replantearon algunas áreas temáticas y nacieron cambios generales en otras. Asimismo, se contó con las contribuciones de Enfermeras especialistas en Terapia Respiratoria y Educación Permanente en Enfermería del Hospital Nacional de Niños, Dr. Carlos Sáenz Herrera: con la contribución de ellas se modificó el manejo específico de procedimientos especiales en el hogar adaptados de manera diferenciada respecto de los procedimientos que se realizan en el nivel intrahospitalario. Con esto se consolidó la guía práctica que sería validada por la población del centro en el que se realizó el diagnóstico.

La segunda fase del proceso de validación correspondió al planteamiento y ejecución de los talleres de validación realizados, siguiendo la base de la guía, en dos sesiones en la Escuela de Rehabilitación y la implementación de un puesto informativo en el cual se tratarían las 
temáticas de manera personalizada. En estas actividades se corroboró que el manejo de las temáticas es el adecuado, los resultados fueron satisfactorios durante el proceso en general. Se evidenció el valor de la aplicación de ayudas visuales como medio facilitador para el entendimiento de las temáticas y la importancia del uso de recordatorios de detalles importantes para reforzarlos.

La calidad de la información, su comprensión y su aceptación -por parte de las y los asistentes a los talleres- y el puesto de información fueron amplias, por ende, quedó justificada la utilidad de la guía en la consecución de los objetivos que se propone concretar.

\section{CONCLUSIONES}

El perfil de las personas
corresponde
cuidadoras a majeres, madres y
principalmente a mujer debe
amas de casa. Lo anterior no debe
ser pasado por alto dado que son
aspectos importantes para abordar a
dicha población. Igualmente, esta
información ya se esperaba dado que
tales labores se encuentran
estereotipadas y se asocian a la
mujer, producto de la socialización
patriarcal.

- El proceso diagnóstico permitió determinar los aspectos referentes al cuidado de los niños, las niñas, los adolescentes y las adolescentes con discapacidad y sirvió como punto de partida para la elaboración de una guía que provee información pertinente para personas que se encuentran en situaciones similares.

- La participación de cuidadores y cuidadoras en los talleres de validación permitió visualizar y rescatar aspectos diarios del cuidado los cuales posibilitaron un mejoramiento de la atención.

- La Enfermería debe ir más allá del suministro de información y debe producir herramientas e instrumentos para que las personas construyan conocimiento y sean capaces de llevar a cabo su autocuidado.

- La falta de acceso a este tipo de información hace -de esta guía práctica- una pionera debido a que ofrece información objetiva y al alcance de todos. De esta manera, la guía práctica diseñada se convierte en un instrumento viable para ser utilizado en el proceso de orientación para brindar el cuidado en el hogar.

- A su vez, a pesar de estar dirigida a una población en específico, la guía práctica puede ser utilizada por profesionales $\mathrm{y}$ estudiantes de diversas áreas en su proceso de formación con el fin de brindar orientación a las personas cuidadoras durante sus intervenciones.

- Además, la atención planteada se sustenta como un instrumento para ser utilizado por el equipo interdisciplinario de la Escuela de Rehabilitación en las visitas al hogar.

\section{BIBLIOGRAFÍA}

Arce, L., Barrientos, R., Castillo, M., y Varela, V. (2006). Intervención de Enfermería Pediátrica en la Escuela de Rehabilitación Nacional. San José, Costa Rica: Universidad de Costa Rica.

Asamblea Legislativa. (1996). Ley 7600 Sobre Igualdad de Oportunidades para las Personas con Discapacidad. Asesoría presidencial para asuntos de la población con discapacidad. San José, Costa Rica. 
Asamblea Legislativa. (1998). Código de la niñez y la adolescencia. Departamento de servicios parlamentarios: área de procesos legislativos. Costa Rica, San José.

Benjumea, C. (2007). El cuidado del otro: Desafíos y posibilidades. Vol.25, $\mathrm{N}^{\mathrm{o}} 1,2007$, pp.106- 112. Consultado el 8 de julio de 2008. www.sicielo.org.co/scielo.php?script=sci_serial \&pid=012-53\&Ing=en\&nrm=iso

Del Águila, A., y Áibar, p. (2006). Características nutricionales de niños con parálisis cerebral. ARIE - Villa El Salvador. Anales de la Facultad de medicina. v.67 n.2 Lima abr.-jun. 2006.

Consultado el 08 de setiembre del 2008. http://www.scielo.org.pe/scielo.php?script=sci_ arttext\&pid=S102555832006000200003\&lng =e s\&nrm=iso>. ISSN 1025-5583.

Espinoza, C., Mena, H., y Salas, M (2004). Calidad de vida de los niños y niñas con discapacidad producida por enfermedad crónica y sus familias, Área de Salud de Montes de Oca. San José, Costa Rica: Universidad de Costa Rica.

Fritz, S. (2001). Fundamentos del masaje terapéutico. Primera Edición. Editorial Paidotribo.

García, M., Mateo, I., y Maroto, G (2004). El impacto de cuidar en la salud y la calidad de vida de las mujeres. Gaceta sanitaria vol.18 suppl.2 Barcelona. Consultado el 20 de junio del 2008. http://www.scielosp.org/scielo.php?script=sci_a rttext\&pid=S02139112004000800011\&lng=en \&nm=iso>.ISSN 0213-9111. doi: 10.1590/S0213-91112004000800011.

Gaite, L., Cantero, P., González, D., y García, M. (2005). Necesidades de los pacientes pediátricos con enfermedades raras y de sus familias en Cantabria. Consultado el 7 de abril del 2007. http://www.cedd.net/publicaciones/coleccion.jsp

Harbin., Behrman., y Kliegman. (1997). Nelson: Tratado de Pediatría. México D.F, México: McGraw-Hill Interamericana Editores, S.A de C.V.

Hernández, R., Fernández-Collado, C., y Baptista, P. (2006). Metodología de la Investigación. México D.F. México: McGrawHill Interamericana Editores, S.A. de C.V.
INEC (2001). IX Censo Nacional de Población y de Vivienda del 2000. Resultados Generales. San José, Costa Rica.

Kozier, B; Erb, G., y Olivieri, R. (1993). Enfermería fundamental: Conceptos, procesos y práctica. Madrid, España: McGraw-Hill Interamericana de España.

Leal, A. (2005). Guía para padres de familia de personas con parálisis cerebral. San José, Costa Rica: Universidad de Costa Rica.

Orem, D. (1983). Normas prácticas en enfermería. Ediciones Pirámide, España.

Organización Mundial de la Salud. (2002) International day of disabled persons: 3 December. Consultado el 03 de julio del 2007. http://www.who.int/mediacentre/news/notes/np 9/en/.

Pampillo, J. (1995). Diagnóstico de necesidades de capacitación de los padres de los niños con discapacidad múltiple y severa de las escuelas de la subregión educativa de San José. San José Costa Rica: Universidad de Costa Rica.

Póo, P. (2008). Parálisis cerebral infantil. Protocolos Diagnóstico Terapéuticos de la Asociación Española de Pediatría: Neurología Pediátrica. Consultado el 25 setiembre de 2008. http://www.aeped.es/protocolos/neurologia/36pci.pdf.

Rodríguez, A. (1995). Escuela para padres, pubertad y adolescencia: La mejor para una relación afectiva y feliz con sus adolescentes. Manual de instrucción. San José, Costa Rica: TOP S.A.

Zambrano, R., Ceballos, P. (2007). Síndrome de carga del cuidador. Revista colombiana de psiquiatría. Vol. 36 supl. 1. p.26-39. Consultado el 02 de julio del 2008. http://www.scielo.org.co/scielo.php?script=sci_ arttext\&pid=S003474502007000500005\&lng =e n\&nrm=iso >. ISSN 0034-7450. 
El euidade en el hogar del niño. la niĥa • adolescente con discapacidad motora

Una guin próetien Jirigida a euidadores y euidadoras

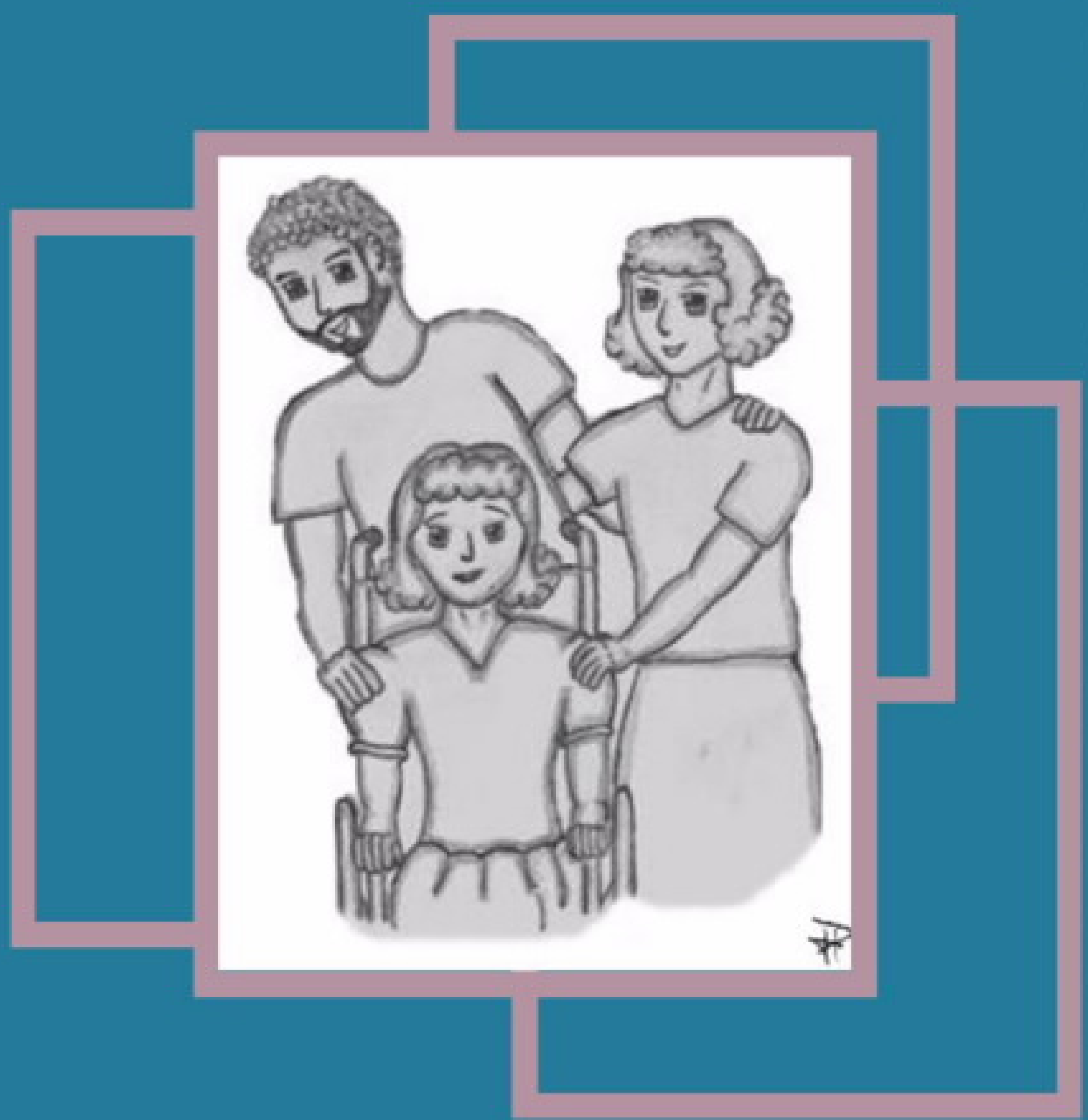

Esteban Mlonso Gareín Leiva Juan Pablo muñoz Campor stephanie Porras Hidalgo 


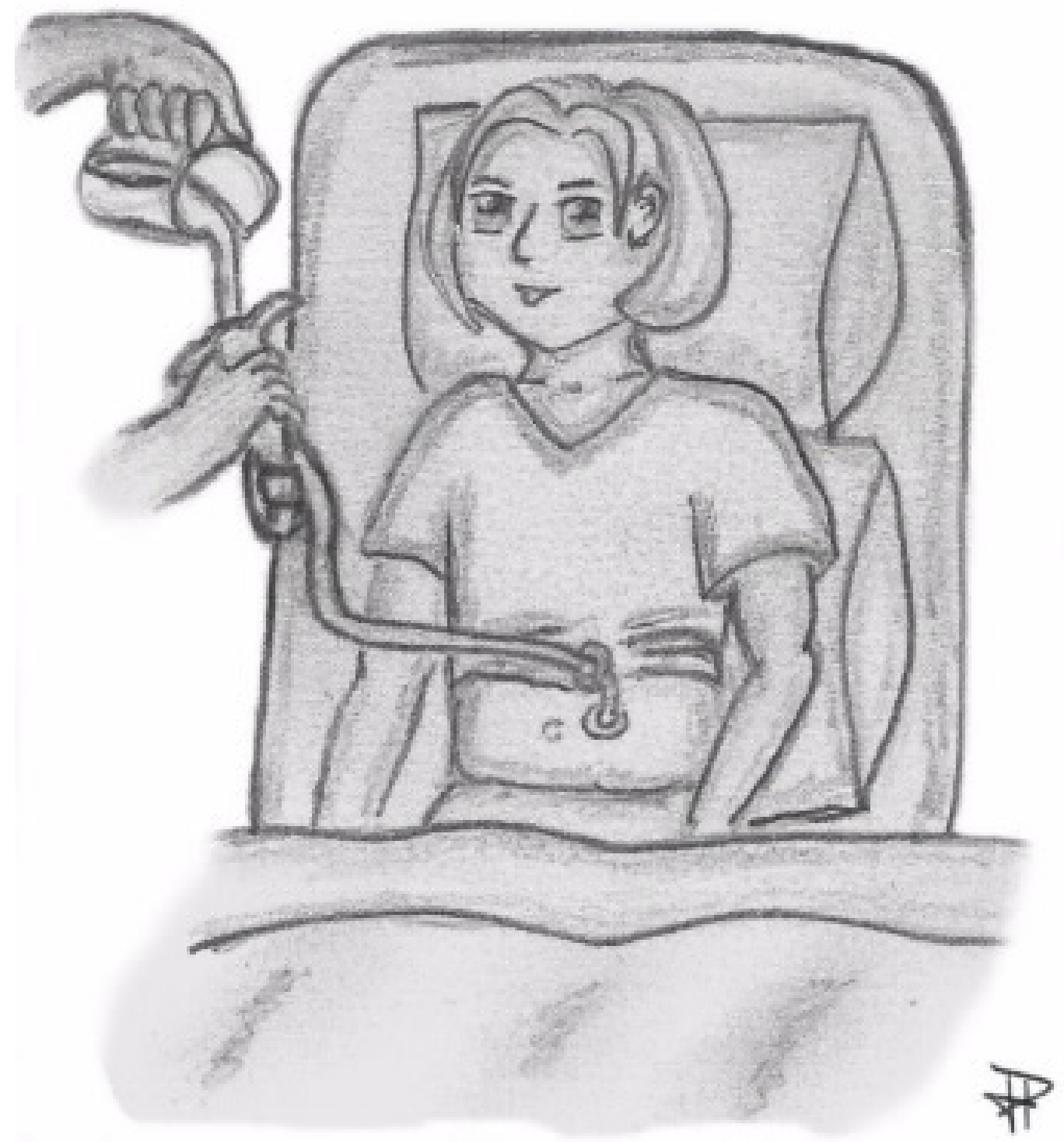




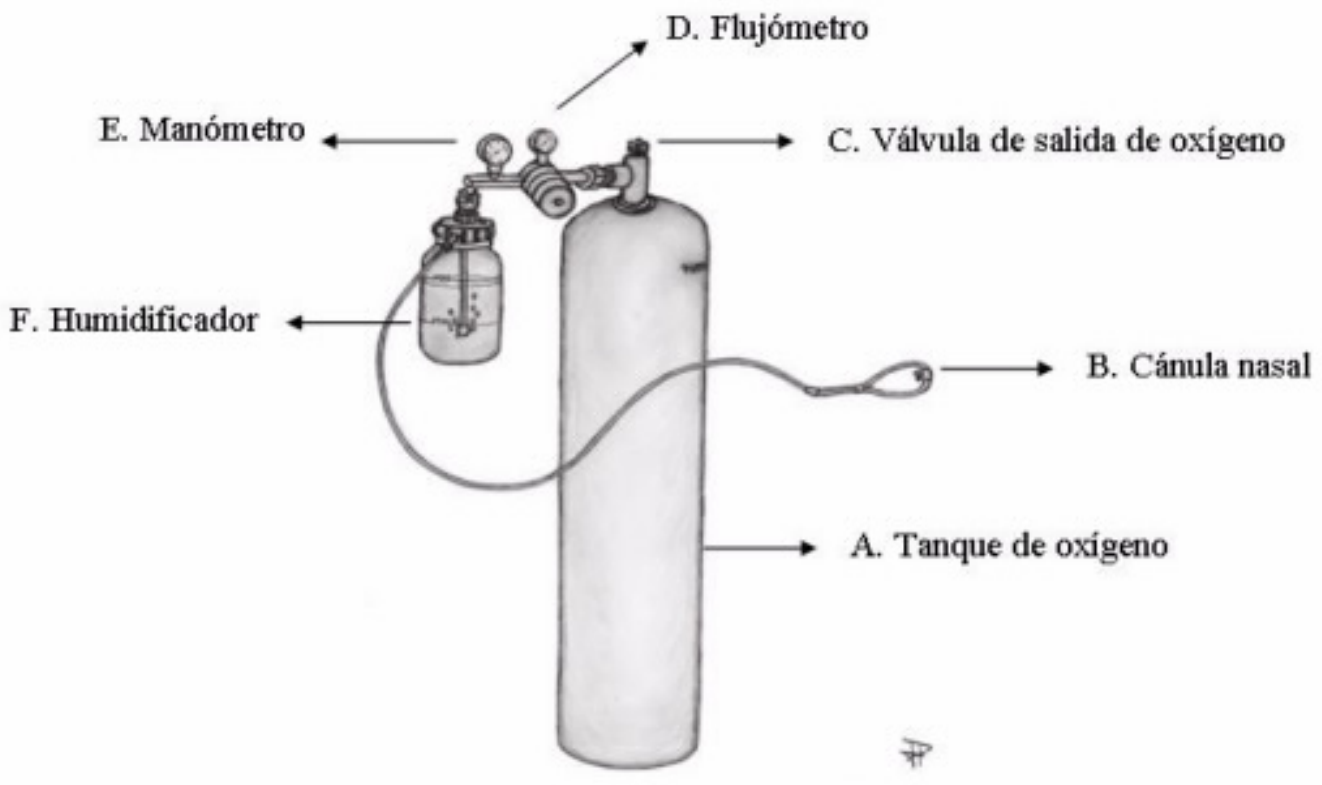

Figura 23. Sistema de administración de oxigeno 


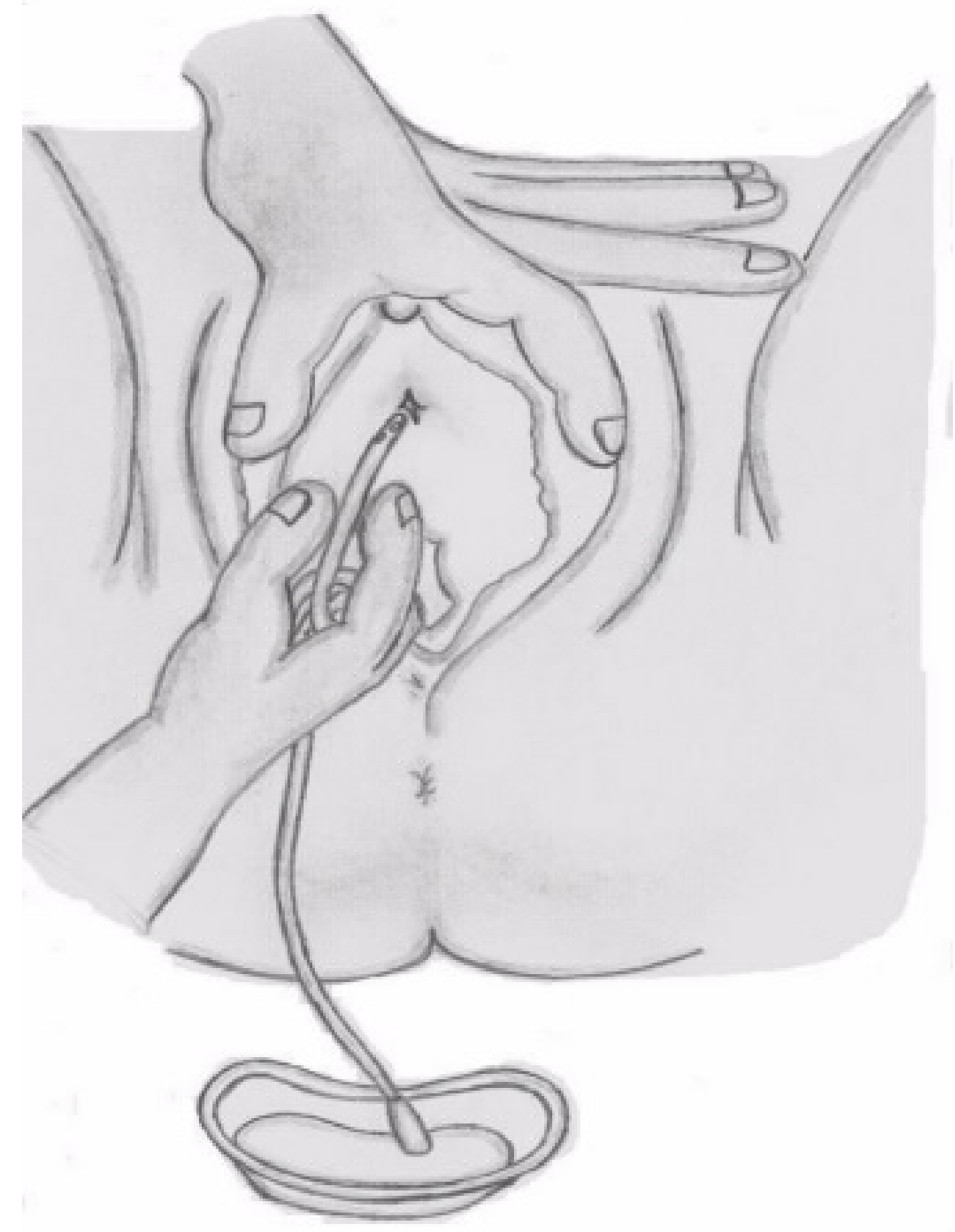


REVENF

Diseño de guía para el cuidado en el hogar de menores con discapacidad motora

17

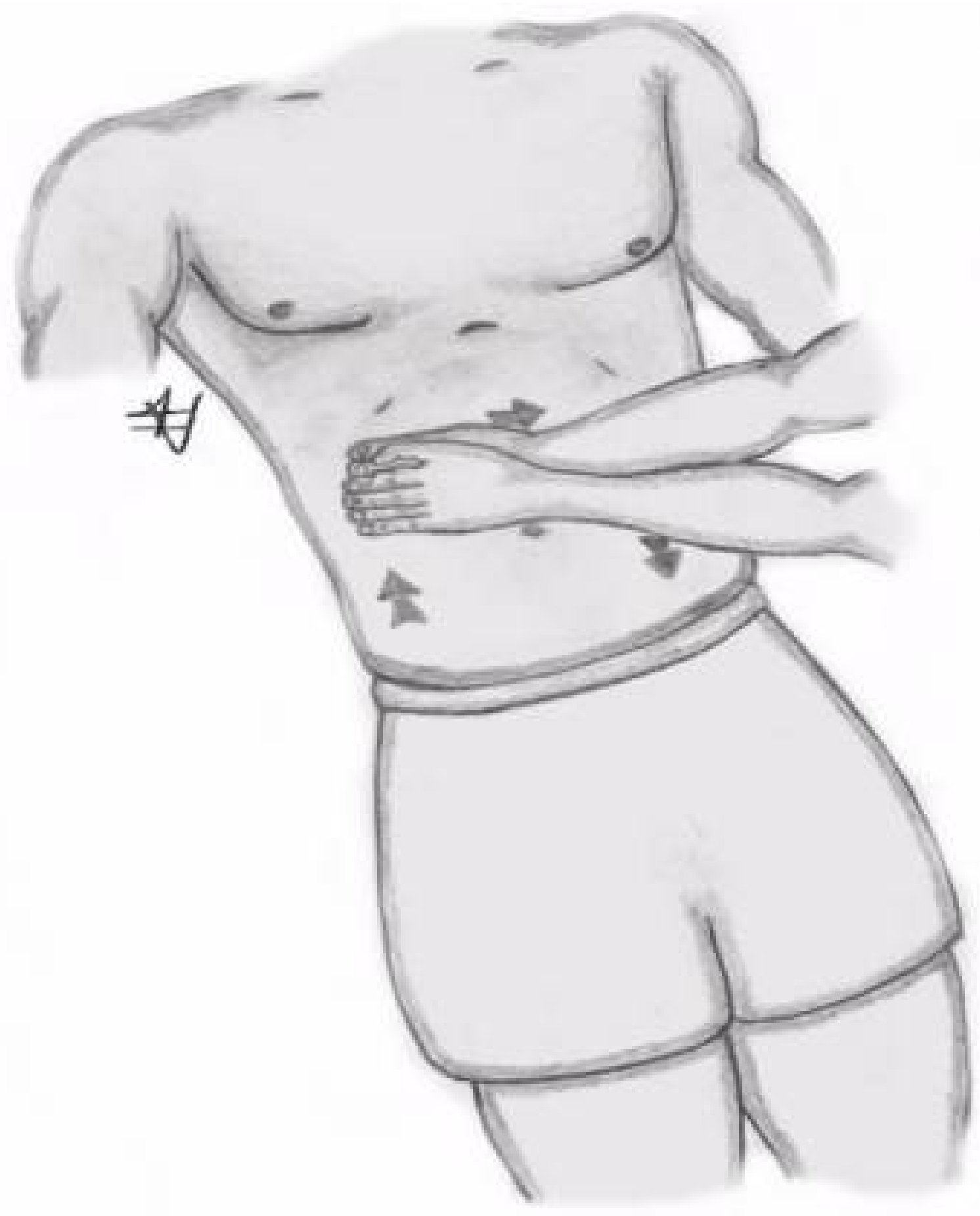




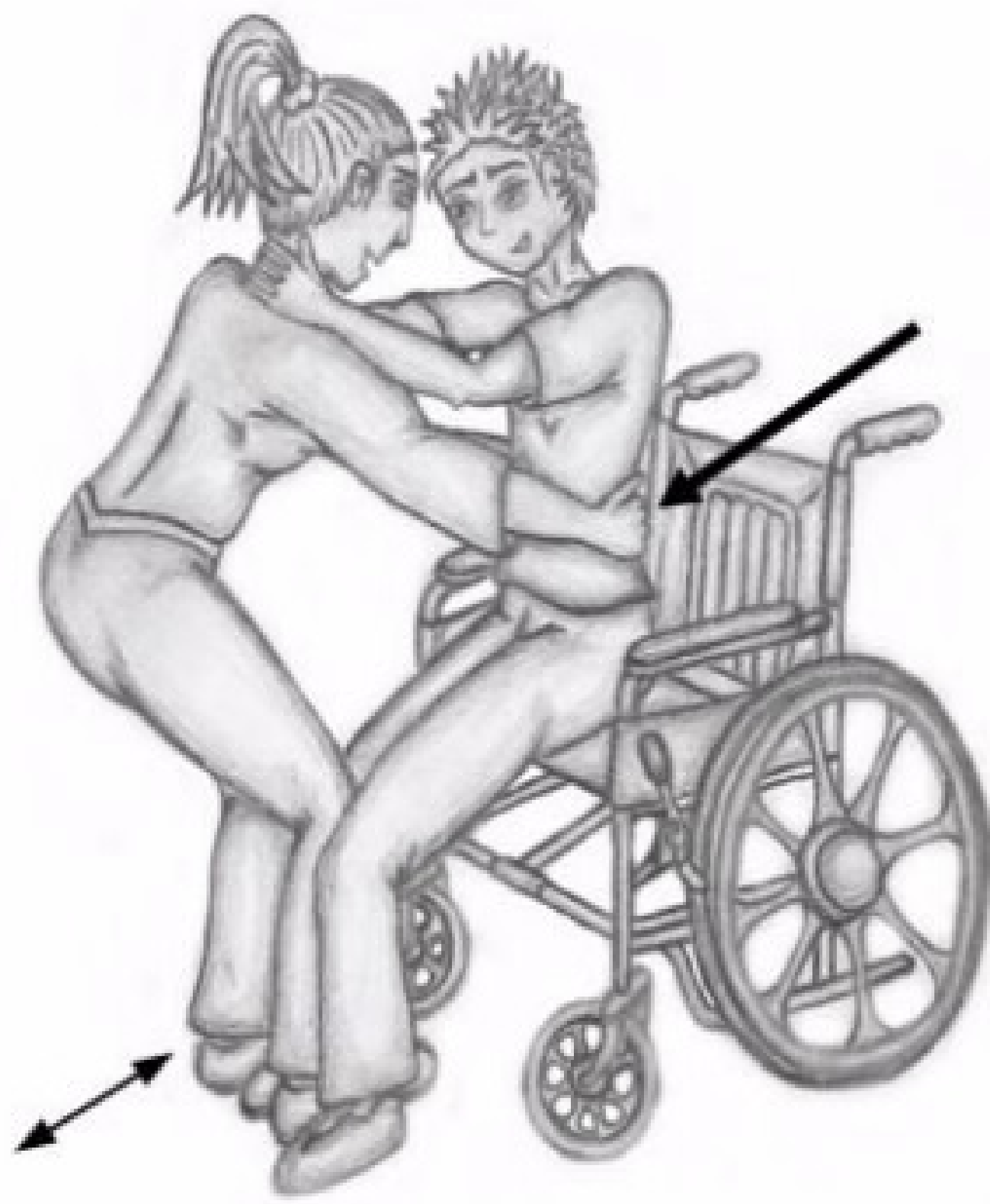




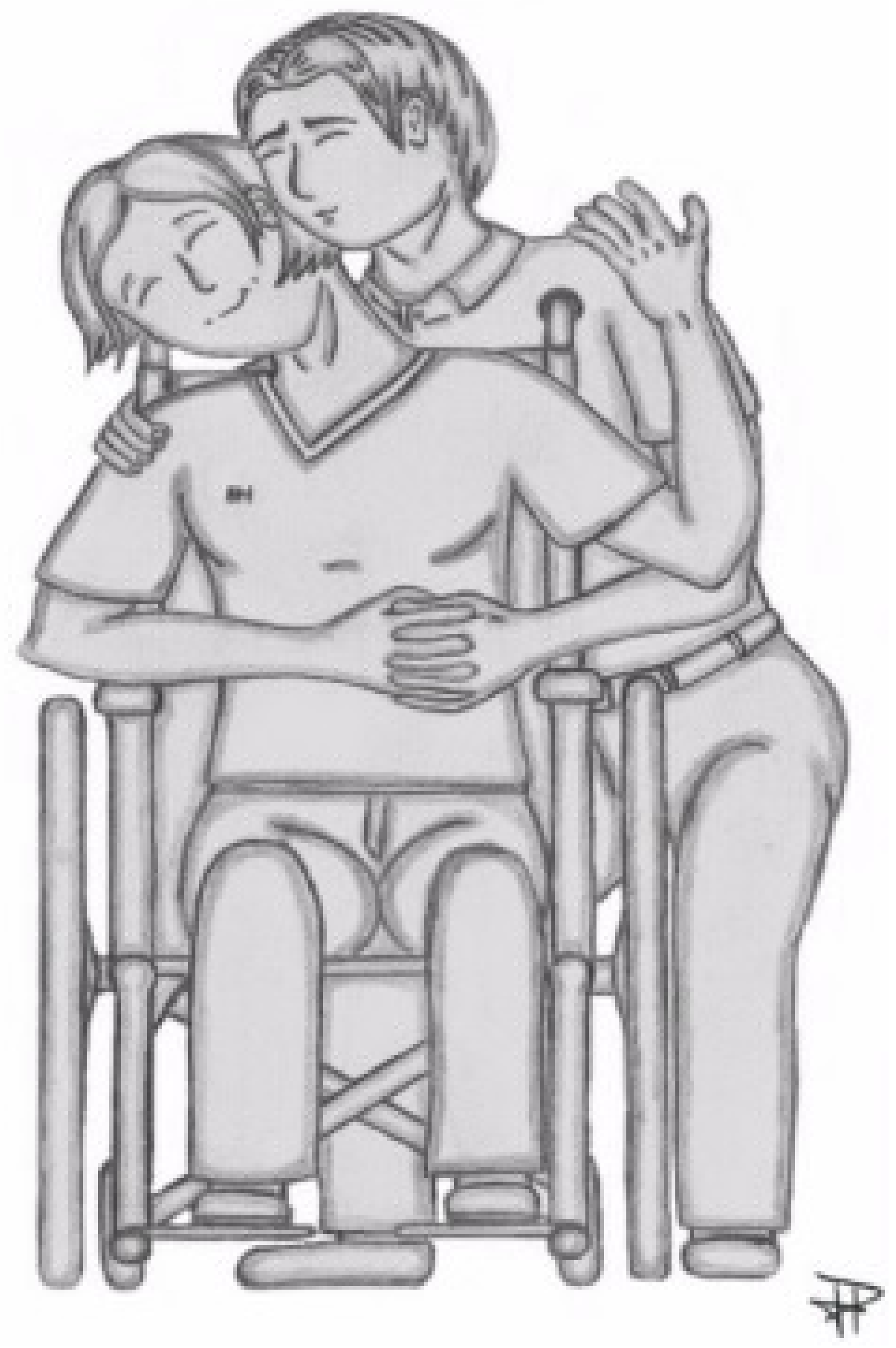




\section{$\mathbf{I}$}

\section{ASPECTOS DEL CUIDADO DEL NIÑO, LA NIÑA, EL ADOLESCENTE O LA ADOLESCENTE CON DISCAPACIDAD MOTORA}

\section{2 * Prevención de Úlcerens por Presión}

¿Qué medidas puedo tomar en cuenta para evitar la aparición de las úlceras por presión en la persona a la que cuído?

\section{Preguntas} introductorias a la temática:

Facilitan la comprensión de la información que se brinda.

- Mantener la piel limpia y seca

- Para limpiar la piel de la persona utilice jabones o sustancia limpiadoras con baja capacidad para producir irritación en la piel.

- Promover que la persona tenga un aporte de líquidos y una nutrición adecuadas.

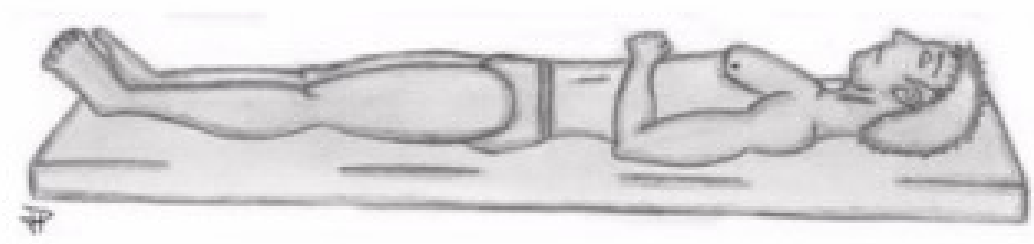

Figura 34. Zonas de Presión 


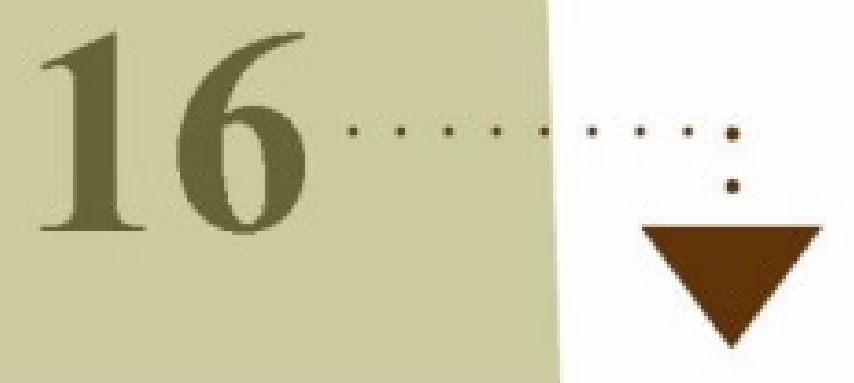

\section{คUTOOUIDADO En El} OUIDADOR 0 in OUIDADORa PRIROIPAI

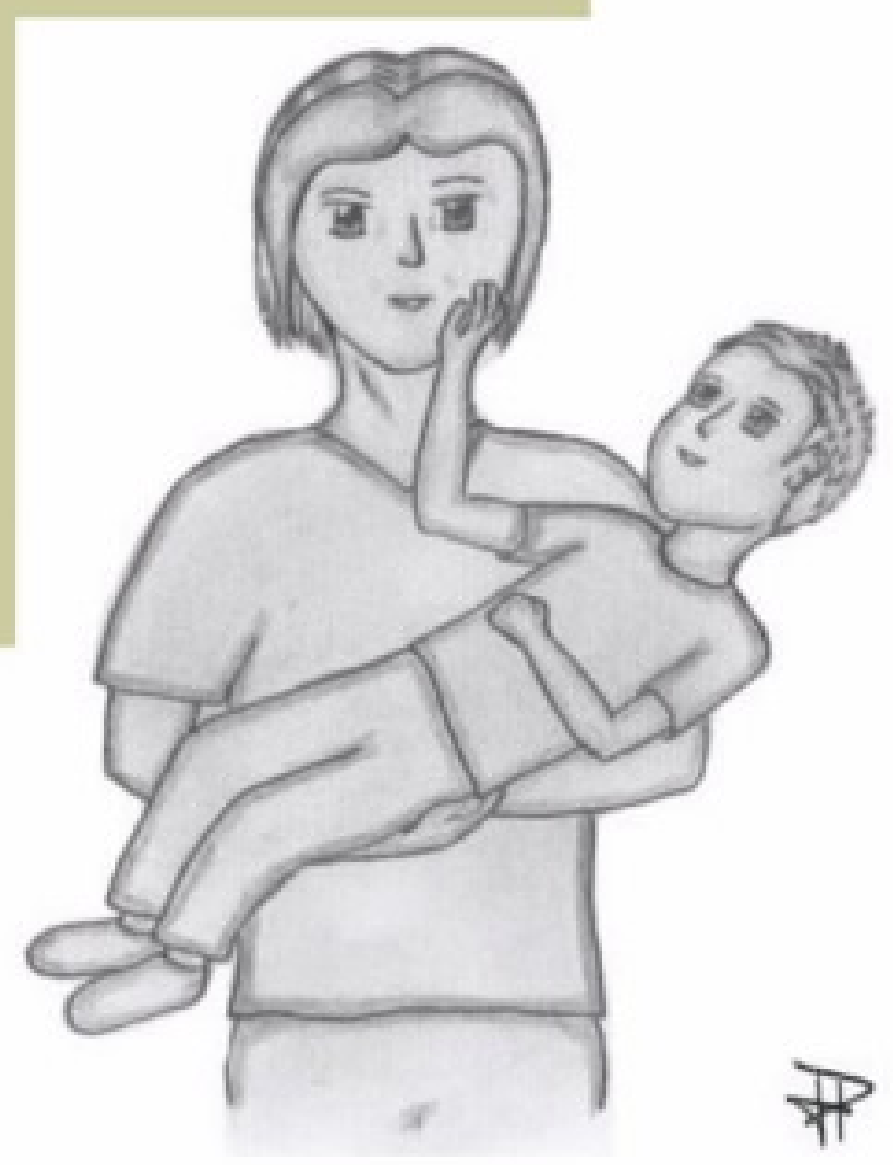




\section{Presentación del Capítulo}

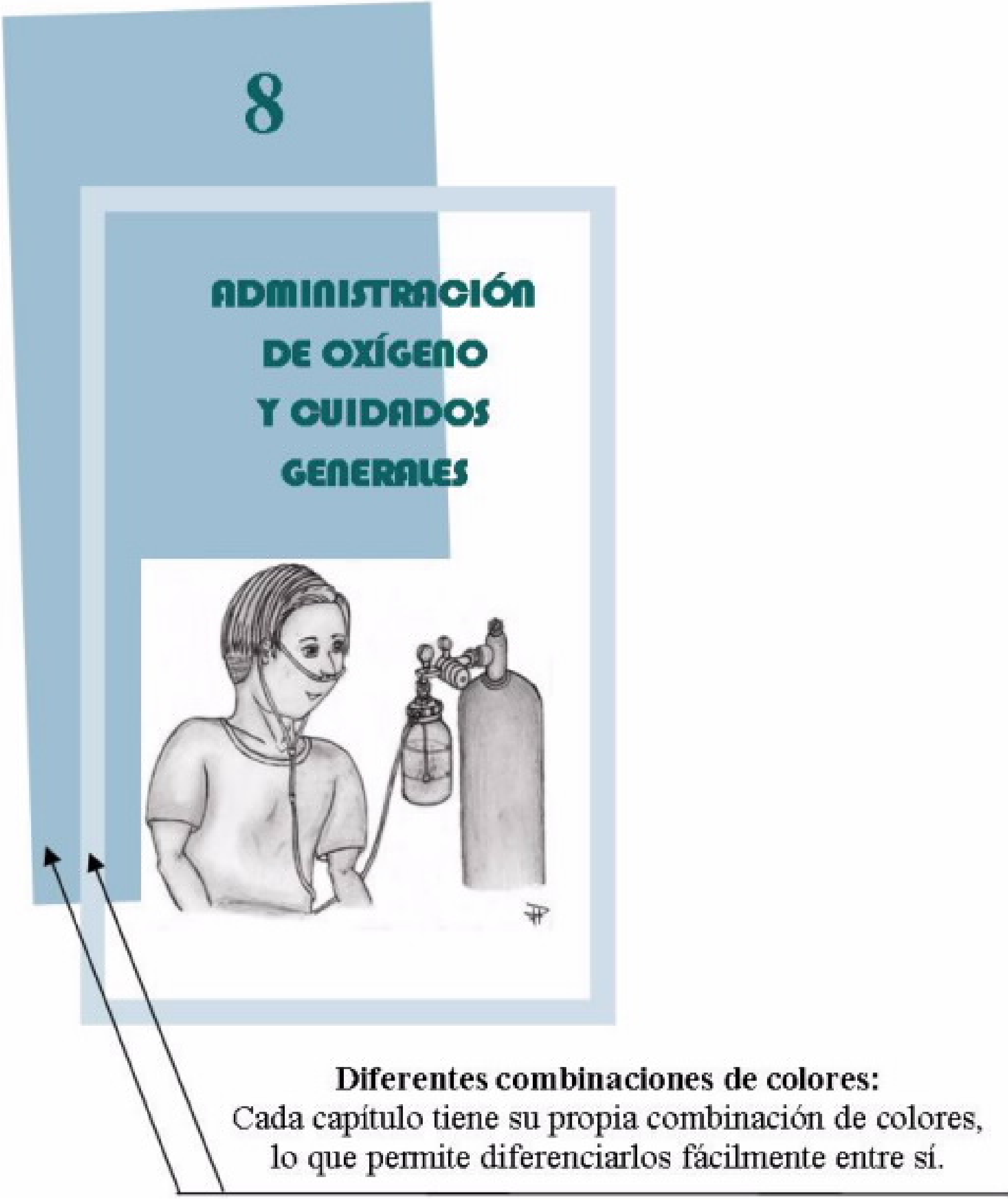

